Proceeding Paper

\title{
Temperature Dependence of Germanium Arsenide Field-Effect Transistors Electrical Properties ${ }^{\dagger}$
}

\author{
Alessandro Grillo 1,2, , Enver Faella 1, Filippo Giubileo 2, Aniello Pelella 1,2, Francesca Urban 1,2 \\ and Antonio Di Bartolomeo ${ }^{1,2, *}$ \\ 1 Physics Department “E. R. Caianiello”, University of Salerno, via Giovanni Paolo II 132, \\ 84084 Fisciano, Salerno, Italy; efaella@unisa.it (E.F.); apelella@unisa.it (A.P.); furban@unisa.it (F.U.) \\ 2 CNR-SPIN Salerno via Giovanni Paolo II 132, 84084 Fisciano, Salerno, Italy; filippo.giubileo@spin.cnr.it \\ * Correspondence: agrillo@unisa.it (A.G.); adibartolomeo@unisa.it (A.D.B.) \\ + Presented at the 2nd International Online-Conference on Nanomaterials, 15-30 November 2020; \\ Available online: https://iocn2020.sciforum.net/.
}

Citation: Grillo, A.; Faella, E.; Giubileo, F.; Pelella, A.; Urban, F.; Di Bartolomeo, A. Temperature Dependence of Germanium Arsenide Field-Effect Transistors Electrical Properties. Mater. Proc. 2021, 4, 26. https://doi.org/10.3390/IOCN202007810

Academic Editors: Ana María Díez-Pascual, Antonio Di Bartolomeo and Guanying Chen

Published: 15 November 2020

Publisher's Note: MDPI stays neutral with regard to jurisdictional claims in published maps and institutional affiliations.

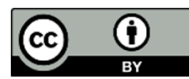

Copyright: () 2020 by the authors. Licensee MDPI, Basel, Switzerland. This article is an open access article distributed under the terms and conditions of the Creative Commons Attribution (CC BY) license (http://creativecommons.org/licenses /by/4.0/).

\begin{abstract}
In this work, we report the fabrication of germanium arsenide (GeAs) field-effect transistors with ultrathin channel and their electrical characterizations in a wide temperature range, from 20 to $280 \mathrm{~K}$. We show that at lower temperatures, the electrical conduction of the GeAs channel is dominated by the 3D variable range hopping but becomes band-type at higher temperatures, after the formation of a highly conducting two-dimensional (2D) channel. The presence of this 2D channel, limited to a few interfacial GeAs layers, is confirmed by the observation of an unexpected peak in the temperature dependence of the carrier density per area at $\sim 75 \mathrm{~K}$. Such a feature is explained considering a model based on a temperature-dependent channel thickness, corroborated by numerical simulations, that show excellent agreement with the experimental data.
\end{abstract}

Keywords: germanium arsenide; 2D conduction; field-effect transistors; carrier concentration; variable range hopping

\section{Introduction}

Recent interest in research on two-dimensional (2D) materials has led to the exploration of new classes of layered materials beyond graphene and transition metal dichalcogenides. Among these, the binary compounds of groups IV and V are assuming considerable importance since theoretical studies have proved their crystalline layered structures with orthorhombic ( $\mathrm{Cmc}_{1}$ for $\mathrm{SiP}$ and $\mathrm{Pbam}$ for $\mathrm{SiP}_{2}$ and $\mathrm{GeAs}_{2}$ ) or monoclinic ( $C 2 / m$ for $G e P, G e A s$, and $S i A s)$ symmetries [1-4]. Particularly, GeAs has been considered for possible optoelectronic applications and for its high in-plane anisotropy [5,6]. Figure 1a shows the GeAs crystal layered structure, in which each Ge atom is bonded to one $G e$ atom and three $A s$ atoms forming distorted $A s_{6} @ G e_{2}$ octahedra [7]. Similar to TMDs, the GeAs bandgap changes according to the number of layers. Numerical calculations and optical bandgap measurements, indicate that germanium arsenide indirect bandgap ranges from $\sim 0.57-0.65 \mathrm{eV}$ for the bulk $[8,9]$ to $1.6-2.1 \mathrm{eV}$ for the monolayer $[10,11]$.

In this work, we report the fabrication of $12 \mathrm{~nm}$ thick GeAs back-gate field effect transistors through mechanical exfoliation and their electrical characterization in a fourprobe configuration and in a range of temperatures from 20 to $280 \mathrm{~K}$. We find that at low temperature, the conduction is dominated by $3 \mathrm{D}$ variable range hopping but becomes band-type at higher temperatures. This change in the conduction mechanism is due to the formation of a highly conducting 2D channel close to the gate, confirmed by the observation of an unexpected peak in the temperature dependence of the carrier density 
per area at $\sim 75 \mathrm{~K}$. The model is corroborated by numerical simulations that show an excellent agreement with the experimental data.

a)
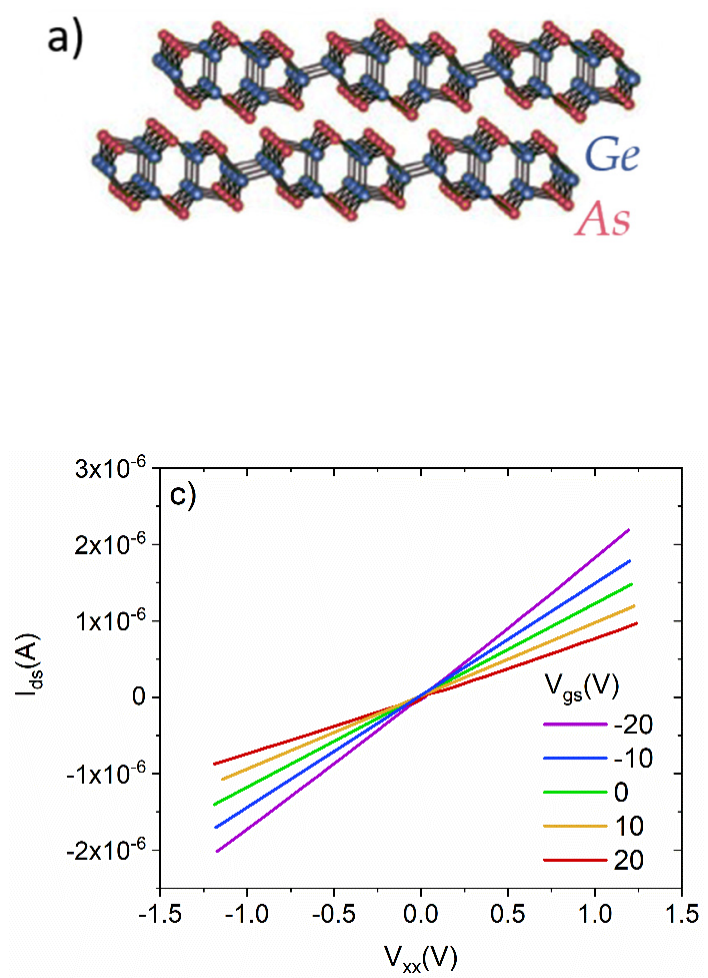
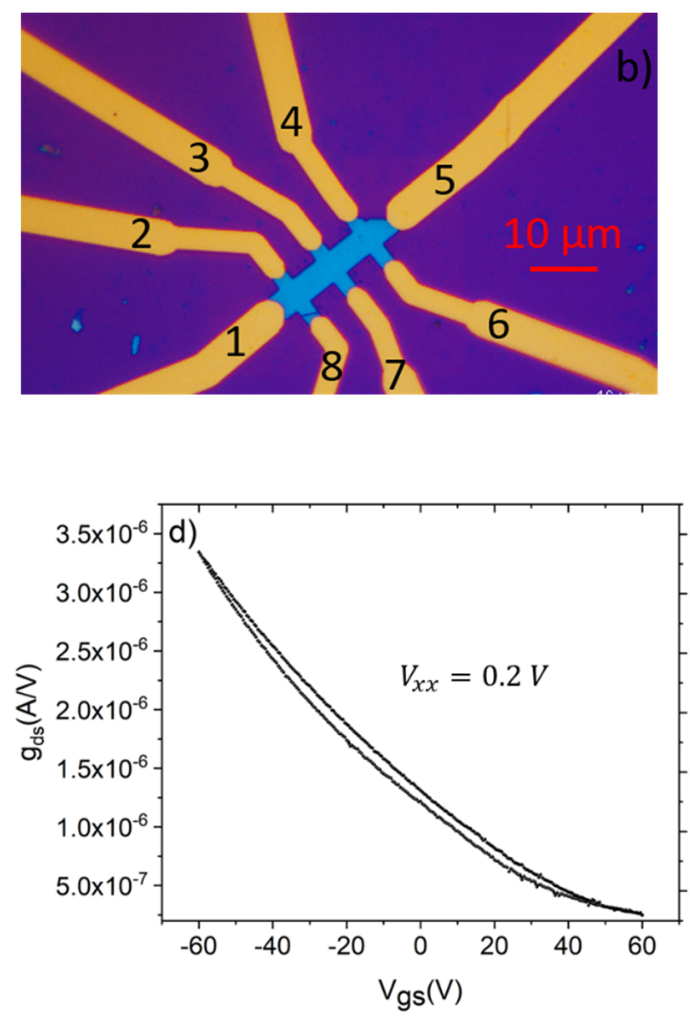

Figure 1. (a) Atom arrangement in GeAs layers. (b) Optical image of the Hall bar device with Ni/Au electrodes. (c) Output characteristic recorded in four-probe configuration with $V_{g s}$ bias between -20 and 20 V. (d) Transfer characteristic recorded in four-probe configuration showed both on linear and logarithmic scale.

\section{Results and Discussion}

Figure $1 \mathrm{~b}$ shows an optical image of a $12 \mathrm{~nm}$ thick GeAs flakes covered with eight $5 \mathrm{~nm} \mathrm{Ni} / 40 \mathrm{~nm} \mathrm{Au}$ electrodes. Ultrathin GeAs flakes were exfoliated from bulk GeAs single crystals using a standard mechanical exfoliation method by adhesive tape. The flakes were transferred onto degenerately doped p-type silicon substrates, covered by $300 \mathrm{~nm}$-thick $\mathrm{SiO}_{2}$. Electron-beam lithography were performed on selected flakes to obtain a Hall bar structure. Finally, electron-beam evaporation was used to deposit the metal electrodes [12].

Contacts 1 to 4 were used to perform a standard four probe electrical characterization in which the current $\left(I_{d s}\right)$ is measured between the outers contact (1 and 4$)$ and the voltage drop $\left(V_{x x}\right)$ is measured between the inner contacts (2 and 3).

Figure 1c shows the output characteristic, i.e., the drain-source current as a function of the voltage drop between two inner contacts with the gate-source voltage $\left(V_{g s}\right)$ as control parameter. It shows a linear behaviour that is not influenced by the gate potential which only changes the total conductance of the device. Moreover, it testifies the formation of ohmic contacts due to the alignment between the Ni Fermi level (work function $5.15 \mathrm{eV}$ ) below the valence band of GeAs. Figure 1d reports the transfer characteristic, i.e., the $g_{d s}-V_{g s}$ curve $\left(g_{d s}=I_{d s} / V_{x x}\right.$ is the channel conductance) measured over a loop of the gate voltage. It shows a typical p-type behaviour mainly due to the presence of intrinsic defects such as $\mathrm{Ge}$ vacancies, the interaction with the $\mathrm{SiO}_{2}$ gate dielectric and the oxidation of the topmost layers of the flake that would act as p-type dopants in the material and provide intragap states [1,2]. 
Figure 2a,b shows the temperature dependence of the electric behaviour of the device in the $20-280 \mathrm{~K}$ temperature range. Particularly, from Figure $2 \mathrm{a}$, we find that the conductance decreases lowering the temperature without any other apparent change in the behavior of the device and from a linear fit of the $I_{d s}-V_{x x}$ curves recorded at $V_{g s}=$ $-20 \mathrm{~V}$, we estimate the resistivity $\rho$ of the sample as a function of the temperature $(\rho=$ $\mathrm{RW} / \mathrm{L}$, where $R$ is the resistance evaluated from the fit and $L=6$ and $W=2.7 \mu \mathrm{m}$ are the channel length and width, respectively). The results of the fit are shown in the inset of Figure $2 \mathrm{a}$ in which a clear semiconductive behavior is observed. Figure $2 \mathrm{~b}$ reports the transfer characteristic of the device in which we defined the FET channel conductance as:

$$
g_{d s}=\frac{I_{d s}}{V_{x x}}=\left(\frac{W}{L}\right) \mu_{F E} C_{o x}\left|V_{g s}-V_{t h}\right|^{\alpha},
$$

where $\mu_{F E}$ is the field-effect mobility; $C_{o x}=\epsilon_{0} \cdot \frac{\epsilon_{\mathrm{SiO}_{2}}}{t_{\mathrm{SiO}_{2}}}=1.15 \cdot 10^{-8} \mathrm{~F} / \mathrm{cm}^{2}$ is the capacitance per unit area of the gate dielectric, where $\epsilon_{0}=8.85 \cdot 10^{-14} \mathrm{~F} / \mathrm{cm}^{2}, \epsilon_{\mathrm{SiO}_{2}}=3.9$, and $t_{\mathrm{SiO}_{2}}=300 \mathrm{~nm}$ are the vacuum permittivity, the $\mathrm{SiO}_{2}$ relative permittivity, and thickness, respectively; $V_{t h}$ is the threshold voltage; and $\alpha \geq 1$ is a dimensionless parameter, which accounts for a possible $V_{g s}$-dependence of the mobility [13]. In order to have a fair comparison with the values of $\rho$ obtained from $g_{d s}-V_{x x}$ curves at $V_{g s}=-20 \mathrm{~V}$, we performed linear fit of the transfer characteristics in a small interval around $V_{g s}=-20 \mathrm{~V}$ in which we can consider $\alpha=1$. From the fit, we can extrapolate the mobility at each temperature as shown in the inset of Figure $2 \mathrm{~b}$. We find a quadratic dependence, $\mu_{F E} \sim T^{2}$, pointing towards a mobility dominated by Coulomb scattering due to ionized impurities [14]. A deeper analysis of the $\rho$ versus $T$ graph, shown in the inset of Figure 2a reveals that the electrical conduction follows two different mechanisms at low and high temperature, respectively. Indeed, we notice that the best fitting of the experimental data at low temperature is obtained using the variable range hopping conduction (VRH). According to VRH theory, the relation between $\rho$ and $T$ can be expressed as [15]:

$$
\rho(T)=\rho_{0} \exp \left(\frac{T_{0}}{T}\right)^{\frac{1}{n+1}},
$$

where $\rho_{0}$ depends on the square root of $T, T_{0}$ is a constant, and $\mathrm{n}$ indicates the dimensionality of the system [16].
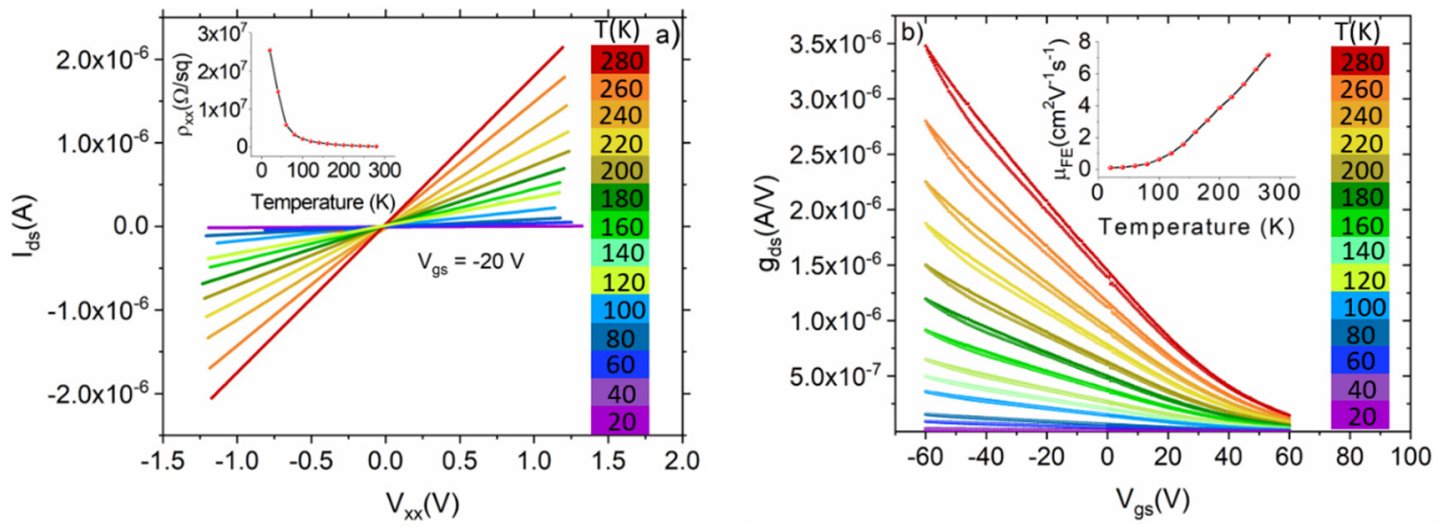

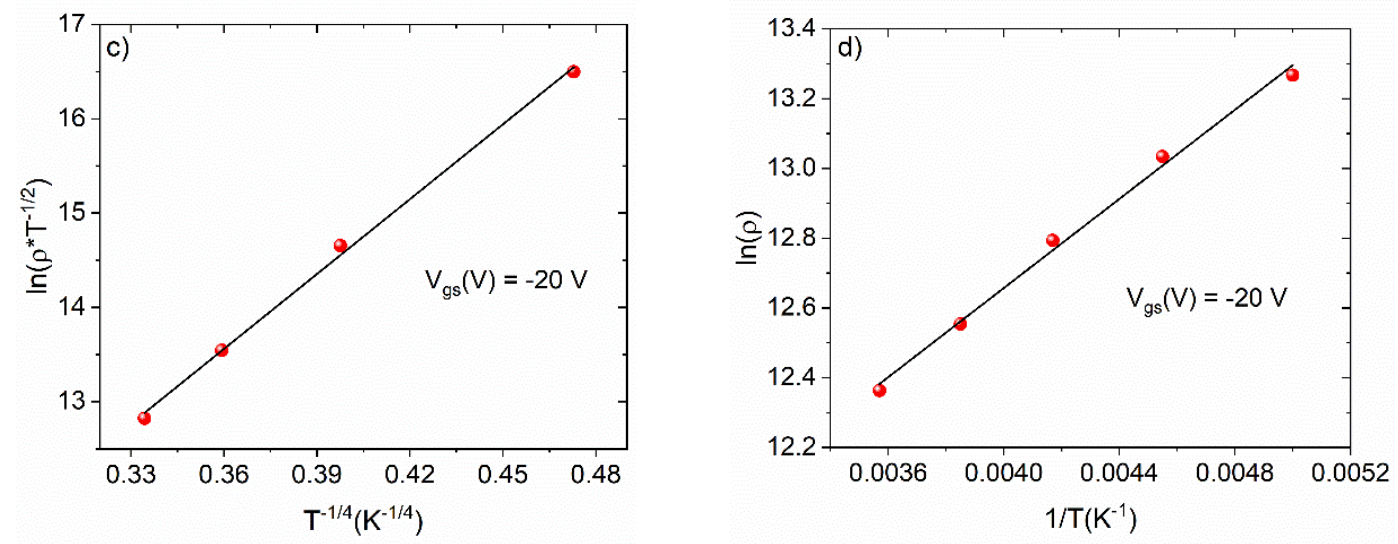

Figure 2. (a) $I_{d s}$ versus $V_{x x}$ curves at different temperatures. The inset shows the channel resistivity as function of the temperature. (b) Transfer characteristics recorded for a loop of the voltage bias at different temperatures. The inset shows the field effect mobility as function of the temperature (c) Experimental data and linear fit of $\ln \left(\rho * T^{-\frac{1}{2}}\right)$ at $V_{g s}=-20 \mathrm{~V}$ as function of $T^{-\frac{1}{4}}$ at low temperature indicating the three-dimensional nature of the investigated system. (d) Experimental data and linear fit of $\ln (\rho)$ at $V_{g s}=-20 \mathrm{~V}$ as function of $T^{-1}$ at high temperature indicating the band conduction regime of the investigated system.

Figure 2c shows $\ln \left(\rho * T^{-\frac{1}{2}}\right)$ as function of $T^{-\frac{1}{4}}$ in the range of $20-80 \mathrm{~K}$. The linearity demonstrates that the conduction is bulk type (3D) for $T<80 \mathrm{~K}$, i.e., it occurs through the entire flake constituted of $\sim 20$ atomic layers. Figure $2 \mathrm{~d}$ shows the trend for $T>$ $180 \mathrm{~K}$. We observe an exponential increase with the inverse of temperature that indicates that the thermal excitation of carriers (holes) to the valence band leads to a band conduction regime, $\rho \propto \exp \left(E_{A} / k T\right)$, as expected after the formation of a highly conductive 2D channel in the GeAs transitor.

In order to try to explain this strange passage from a bulk to a $2 \mathrm{D}$ conduction, we calculate the carrier density per unit area $n\left(\right.$ in $\left.\mathrm{cm}^{-2}\right)$ as a function of the temperature. Indeed, having computed $\mu_{F E}(T)$ and $\rho(T)$ at each of the considered temperatures, $n$ can be obtained from the relation:

$$
n(T)=1 /\left(q \rho(T) \mu_{F E}(T)\right)
$$

where $q$ is the electron charge. Figure 3a shows that $n(T)$ exhibits an unexpected and pronounced peak at $T \sim 75 \mathrm{~K}$, followed by a smoother increase for $T>150 \mathrm{~K}$. In a doped three-dimensional semiconductor, the carrier density would show a different temperature behavior [14]. Specifically, an initial rapid increase due to the ionization of dopant atoms by thermal energy (freeze-out region, up to $\sim 150 \mathrm{~K}$ for medium-doped $\mathrm{Si}$ ) would be followed by a plateau over a wide temperature range when complete ionization is reached (extrinsic region, $\sim 150-500 \mathrm{~K}$ for medium-doped $\mathrm{Si}$ ), and would finally evolve in an exponential increase at higher temperatures due to intrinsic generation of electron-hole pairs (intrinsic region). To explain both the reported anomalous behavior and the modification from a bulk to a 2D conduction due to increasing temperature, we propose a model that considers a temperature-dependent thickness of the channel in which most of the conduction occurs. Indeed, we notice that the 2D carrier density per unit area can be expressed as $n(T)=\varphi(T) \tau(T)$ where $\varphi$ is the carrier density per unit volume in $\mathrm{cm}^{-3}$ and $\tau$ is the thickness of the conducting channel. The current in the GeAs flake is due to carriers injected from the $\mathrm{Ni}$ contacts that overcome a potential barrier, $E_{A}$, between the contacts and the channel region. Therefore, $\varphi(T)$ in the region between the inner contacts can be expressed as [17]: 


$$
\varphi(T)=A \exp \left[-E_{A} / k T\right]
$$

where $k$ is the Boltzmann constant and $A$ is a proportionality constant that can be considered as a fitting parameter of the model.
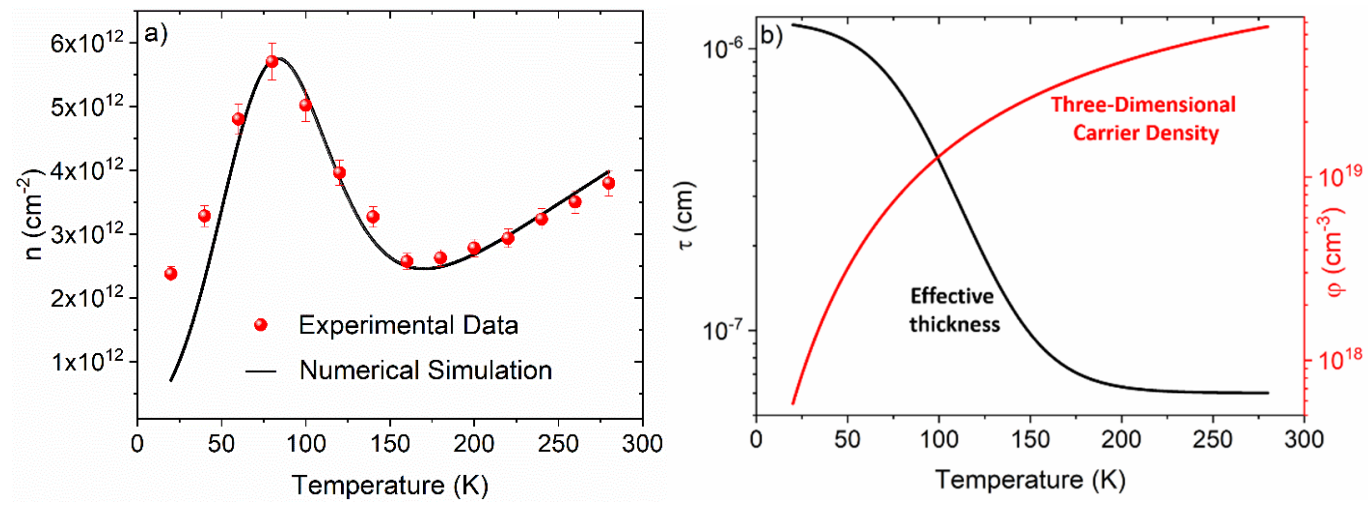

Figure 3. (a) Numerical simulation of the $2 \mathrm{D}$ carrier density as function of the temperature for $V_{g s}=-20 \mathrm{~V}$ superimposed to the experimental data (red dots). (b) Effective thickness of the channel (black line) and three-dimensional carrier density (red line) as function of the temperature for $V_{g s}=-20 \mathrm{~V}$.

At low temperature $(<80 \mathrm{~K})$, there is a limited amount of carriers as well as high intralayer resistance that suppresses the vertical transport with respect to the in-plane one $[18,19]$. In this regime, conduction occurs mainly through the layers in closer contact with the metal leads (especially the topmost ones) and the gate electric field controls the entire flake thickness. With raising temperature, defect ionization, and injection from the contacts increase. The new available carriers, pushed by the applied gate voltage and the favorable vertical band bending toward the interface with the gate dielectric, form a $2 \mathrm{D}$ channel, which becomes more and more conductive with the rising temperature. This channel now screens the gate field, and any variation of the gate voltage affects mainly the bottommost layers of the flake. In this regime, the conduction is dominated by a few atomic $\mathrm{GeAs}$ layers closer to the gate, i.e., the effective conducting thickness $\tau(T)$ controlled by the gate is reduced.

To test the model, with an appropriate choice of $\varphi(T)$ and $\tau(T)$, we computed $n(T)$ obtaining an excellent agreement with the experimental data, as shown in Figure 3a, where we display $n(T)$ at $V_{g s}=-20 \mathrm{~V}$. The carrier density per volume $\varphi(T)$, given by Equation (4), is shown by the red line of Figure $3 \mathrm{~b}$, with $E_{A}$ obtained as fitting parameter. For $\tau(T)$, we used a step-like function, $\tau(T)=A /(1+\exp (B * T))+C$, represented by the black curve shown in Figure $3 b$, in which $A$ is the initial thickness of the whole flake, i.e., $\sim 12 \mathrm{~nm}, C$ is the thickness of a single GeAs layer $(0.6 \mathrm{~nm})$, and $B$ is a fitting parameter used to simulate a smooth transition in the $70-150 \mathrm{~K}$ range. Finally, the model is further corroborated by the estimation of the Debye screening length $L_{D}=\sqrt{\frac{\epsilon \epsilon_{0} k T}{q^{2} \varphi}}$, that gives the length over which the electric field strength drops by a factor 1/e (here $\epsilon=8$ is the dielectric constant at room temperature of the material [20]). From our data, we estimate $L_{D} \sim 0.4 \mathrm{~nm}$ at room temperature, confirming that the gate electric field is substantially screened in the layer closer to the gate.

\section{Conclusions}

We fabricated back-gate field effect transistors with ultrathin GeAs films and investigated their electrical properties over a wide temperature range, from 20 to $280 \mathrm{~K}$. We found a p-type behaviour with temperature increasing conductivity. We find that the con- 
duction is dominated by the $3 \mathrm{D}$ variable range hopping at lower temperatures but becomes band-type at higher temperatures, when a highly conducting 2D channel is formed. We observed that the carrier density in GeAs flakes depends on temperature with a pronounced and broad peak around $75 \mathrm{~K}$. We proposed a model based on a temperaturedependent channel thickness to explain such an anomaly that shows an excellent agreement with the experimental data.

Institutional Review Board Statement: Not applicable.

Informed Consent Statement: Not applicable.

Data Availability Statement: Not applicable.

\section{References}

1. Wu, P.; Huang, M. Stability, bonding, and electronic properties of silicon and germanium arsenides: Stability, bonding, and electronic properties of Si and Ge arsenides. Phys. Status Solidi (b) 2016, 253, 862-867, doi:10.1002/pssb.201552598.

2. Jung, C.S.; Kim, D.; Cha, S.; Myung, Y.; Shojaei, F.; Abbas, H.G.; Lee, J.A.; Cha, E.H.; Park, J.; Kang, H.S. Two-dimensional GeAs with a visible range band gap. J. Mater. Chem. A 2018, 6, 9089-9098, doi:10.1039/C8TA02676A.

3. Cheng, A.-Q.; He, Z.; Zhao, J.; Zeng, H.; Chen, R.-S. Monolayered Silicon and Germanium Monopnictide Semiconductors: Excellent Stability, High Absorbance, and Strain Engineering of Electronic Properties. ACS Appl. Mater. Interfaces 2018, 10, 51335139, doi:10.1021/acsami.7b17560.

4. Shojaei, F.; Kang, H.S. Electronic Structures and Li-Diffusion Properties of Group IV-V Layered Materials: Hexagonal Germanium Phosphide and Germanium Arsenide. J. Phys. Chem. C 2016, 120, 23842-23850, doi:10.1021/acs.jpcc.6b07903.

5. Guo, J.; Liu, Y.; Ma, Y.; Zhu, E.; Lee, S.; Lu, Z.; Zhao, Z.; Xu, C.; Lee, S.-J.; Wu, H.; et al. Few-Layer GeAs Field-Effect Transistors and Infrared Photodetectors. Adv. Mater. 2018, 30, 1705934, doi:10.1002/adma.201705934.

6. Yang, S.; Yang, Y.; Wu, M.; Hu, C.; Shen, W.; Gong, Y.; Huang, L.; Jiang, C.; Zhang, Y.; Ajayan, P.M. Highly In-Plane Optical and Electrical Anisotropy of 2D Germanium Arsenide. Adv. Funct. Mater. 2018, 28, 1707379, doi:10.1002/adfm.201707379.

7. Blachnik, R.; Irle, E. Das System Gallium-Tellur. J. Less Common Met. 1985, 113, L1-L3, doi:10.1016/0022-5088(85)90158-4.

8. Lee, K.; Kamali, S.; Ericsson, T.; Bellard, M.; Kovnir, K. GeAs: Highly Anisotropic van der Waals Thermoelectric Material. Chem. Mater. 2016, 28, 2776-2785, doi:10.1021/acs.chemmater.6b00567.

9. Rau, J.W.; Kannewurf, C.R. Optical Absorption, Reflectivity, and Electrical Conductivity in GeAs and GeAs2. Phys. Rev. B 1971, 3, 2581-2587, doi:10.1103/PhysRevB.3.2581.

10. Zhou, L.; Guo, Y.; Zhao, J. GeAs and SiAs monolayers: Novel 2D semiconductors with suitable band structures. Phys. E LowDimens. Syst. Nanostruct. 2018, 95, 149-153, doi:10.1016/j.physe.2017.08.016.

11. Mortazavi, B.; Shahrokhi, M.; Cuniberti, G.; Zhuang, X. Two-Dimensional SiP, SiAs, GeP and GeAs as Promising Candidates for Photocatalytic Applications. Coatings 2019, 9, 522, doi:10.3390/coatings9080522.

12. Grillo, A.; Di Bartolomeo, A.; Urban, F.; Passacantando, M.; Caridad, J.M.; Sun, J.; Camilli, L. Observation of 2D Conduction in Ultrathin Germanium Arsenide Field-Effect Transistors. ACS Appl. Mater. Interfaces 2020, 12, 12998-13004, doi:10.1021/acsami.0c00348.

13. Di Bartolomeo, A.; Genovese, L.; Giubileo, F.; Iemmo, L.; Luongo, G.; Foller, T.; Schleberger, M. Hysteresis in the transfer characteristics of MoS 2 transistors. 2D Mater. 2017, 5, 015014, doi:10.1088/2053-1583/aa91a7.

14. Sze, S.M.; Ng, K.K. Physics of Semiconductor Devices, 3rd ed.; Wiley-Interscience: Hoboken, NJ, USA, 2007; ISBN 978-0-471-143239.

15. Mott, N.F. Conduction in glasses containing transition metal ions. J. Non-Cryst. Solids 1968, 1, 1-17, doi:10.1016/00223093(68)90002-1.

16. Barreteau, C.; Michon, B.; Besnard, C.; Giannini, E. High-pressure melt growth and transport properties of SiP, SiAs, GeP, and GeAs 2D layered semiconductors. J. Cryst. Growth 2016, 443, 75-80, doi:10.1016/j.jcrysgro.2016.03.019.

17. Ang, Y.S.; Yang, H.Y.; Ang, L.K. Universal Scaling Laws in Schottky Heterostructures Based on Two-Dimensional Materials. Phys. Rev. Lett. 2018, 121, 056802, doi:10.1103/PhysRevLett.121.056802.

18. Liu, Y.; Guo, J.; He, Q.; Wu, H.; Cheng, H.-C.; Ding, M.; Shakir, I.; Gambin, V.; Huang, Y.; Duan, X. Vertical Charge Transport and Negative Transconductance in Multilayer Molybdenum Disulfides. Nano Lett. 2017, 17, 5495-5501, doi:10.1021/acs.nanolett.7b02161.

19. Ji, H.; Ghimire, M.K.; Lee, G.; Yi, H.; Sakong, W.; Gul, H.Z.; Yun, Y.; Jiang, J.; Kim, J.; Joo, M.-K.; et al. Temperature-Dependent Opacity of the Gate Field Inside MoS2 Field-Effect Transistors. ACS Appl. Mater. Interfaces 2019, 11, 29022-29028, doi:10.1021/acsami.9b06715.

20. Mead, D.G. Long wavelength study of semiconducting germanium arsenide, GeAs. Infrared Phys. 1982, 22, 209-213, doi:10.1016/0020-0891(82)90045-8. 